(Aus dem Pathologischen Institut der deutschen Universität in Prag [Vorstand: A. Ghon].)

\title{
Ein Beitrag zu den Teratomen der Brusthöhle.
}

\author{
Von \\ Assist. Dr. Kornel Terplan. \\ (Eingegangen am 30. Juni 1922.)
}

Die Teratome der Brusthöhle sind dem Kliniker wie dem pathologischen Anatomen gut bekannt. Die älteste Mitteilung über ,eine Balggeschwulst" oberhalb des Sternums und des linken Sternoclaviculargelenkes stammt von Gordon, einem englischen Autor, aus dem Jahre 1823.

Seither sind zahlreiche weitere Fälle, teils genau, teils kurz beschrieben worden. Hertzell berichtet bis zum Jahre 1917 über 72 Fälle. Ich konnte aus der mir zur Verfügung stehenden Literatur bis 1920 nur in 68 Fälle Einsicht nehmen. Die meisten Mitteilungen, besonders die größeren zusammenfassenden Arbeiten stammen von klinischer, speziell chirurgischer Seite, so besonders die der letzten Jahre, in denen mehrfach von erfolgreich exstirpierten Teratomen des Mediastinums berichtet wird.

Auch in morphologischer Beziehung verdienen die Mediastinalteratome heute noch unser Interesse, um so mehr als unter den zahlreichen Fällen nur wenige histologisch genau untersucht worden sind, und bei der daraus resultierenden Verschiedenheit der Befunde eine einheitliche Deutung erschwert erscheint. Ungeklärt ist noch immer die Frage nach der Genese der Mediastinalteratome, die nur in Abhängigkeit von Aufbau und Bestandteilen des Teratoms zu lösen ist. Doch ist sie letzten Endes eine Teilfrage des Teratomproblems überhaupt und kann gerecht nur von diesem höheren Gesichtspunkte aus beurteilt werden.

Bevor ich einen klinisch wie pathologisch-anatomisch bemerkenswerten Fall beschreibe, der in unserem Institut zur Beobachtung kam, möchte ich aus der Literatur in Kürze das Wesentliche über Klinik, Morphologie und Genese der Mediastinalteratome herausgreifen.

Sie finden sich bei beiden Geschlechtern und in jedem Alter, doch in überwiegender Zahl in mehr jugendlichem Alter, im zweiten bis vierten Jahrzehnt. Ihr Sitz und wahrscheinlicher Ausgangsort ist für gewöhnlich das vordere Mediastinum, von dem aus sie je nach ihrer 
Größe auch in die linke oder rechte Pleurahöhle hineinwachsen. Bei großen Teratomen, die etwa eine ganze Pleurahöhle einnehmen und sich auch in sagittaler Richtung vom Sternum bis zur Wirbelsäule ausdehnen, ist es wohl kaum mit Sicherheit zu entscheiden, ob ihr Aus* gangsort das vordere oder hintere Mediastinum ist. Die meisten Mediastinalteratome liegen im Spatium mediast, anterius, sitzen dem Herzbeutel oder den großen Gefäßen auf oder liegen zwischen diesen und einer der angrenzenden medialen Lungenfläche. Thre Größe schwankt zwischen der eines Taubeneies bis zu Mannskopfgröße. Die meisten sind gänseei- bis faust- und kindskopfgroß. Doch sind auch riesige „,Dermoidcysten" von $6300 \mathrm{~g}$, sogar von $11 \mathrm{~kg}$ Gewicht bekannt geworden. Sie enthalten im übrigen teils eine einzige große, teils mehrere kleine cystische Räume, zwischen denen mitunter in verschiedener Ausbreitung und Dicke solide Partien liegen.

Im engsten Zusammenhang mit ihrer Größe und Ausdehnung stehen die Lagebeziehungen zu den Organen der Brusthöhle, zu Herz, Lungen, Perikard, Pleuren, Bronchien und zu den großen Gefäßen. Verwachsungen der Cystenwand mit Herzbeutel und Lungen werden vielfach erwähnt, ebenso Verdrängungserscheinungen an den Nachbarorganen: so Kompressionsatelektasen der Lungen, Verlagerung des Herzens und der großen Gefäße, Kompression der großen Venen mit Thrombosen. Auch echte entzündliche Vorgänge spielen sich an den nahen, teilweise mit der Cyste verwachsenen serösen Häuten $\mathbf{a b}$; so werden mehrfach serös-eitrige Entzündungen der Pleuren und seröshämorrhagische Entzündungen des Perikards erwähnt, wobei dann diese sekundären Veränderungen oft das klinische Bild beherrschen.

In einer verhältnismäßig großen Anzahl von Fällen bestand eine Verbindung des Cystenlumens mit den Luftwegen und dementsprechend Auswurf von Haaren oder Talg und Cholesterinmassen, ein Symptom, das diagnostisch von ausschlaggebender Bedeutung war. Des weiteren wird einmal eine Verbindung des Cystenlumens mit der Aorta erwähnt und ebenso eine mit der Perikardhöhle.

Die Diagnose wurde meistens erst intra operationem gestellt, wenn nicht Auswrurf von Haaren oder Dermoidbrei vorausgegangen war; und nur in der letzten Zeit sind einige wenige Fälle bekannt geworden, die mit Hilfe der Röntgendurehleuchtung erkannt worden waren. Unter den Vermutungsdiagnosen wird schon bei einem der ältesten, von Pöhn mitgeteilten Fall Aneurysma der Aorta genannt; im übrigen Pleura- und Perikardergüsse, retrosternale Strumen und Mediastinaltumoren.

Die Therapie ist eine möglichst radikale. Die frühzeitige. Total. exstirpation gilt als Methode der Wahl; die älteren Behandlungsverfahren bestanden in der Eröffnung der Cyste, Drainage, Verschorfung 
oder Ätzung der Innenwand oder in Entfernung von Teilstücken der Cystenwand.

Was die gewebliche Zusammensetzung der Mediastinalteratome betrifft, so möchte ich unter ihnen eine große Gruppe absondern, die mehr als die Hälfte aller bekannten Fälle betrifft; sie enthalten neben mesenchymalen Produkten - Bindegewebe, Knorpel oder Knochen - ausschließlich Elemente der Haut. Sie werden in der Literatur auch als einfache Dermoidcysten benannt. Ihr Lumen enthält den typischen Dermoidbrei, mit Haaren, manchmal Cholesterinmassen, ihre Wand Haut und Hautdrüsen. Doch ist eine häutige Auskleidung vielfach nicht mehr vorhanden. Es muß betont werden, daß dem Versuch einer solchen Gruppierung der Mediastinalteratome etwas Gewaltsames anhaftet, da wohl die Mehrzahl dieser sogenannten Dermoidcysten nicht so genau untersucht worden ist, wie das gerade bei allen Teratomen oder teratomähnlichen Bildungen notwendig wäre.

Neben Derivaten der Haut wird in drei Fällen von den übrigen sogenannten einfachen Dermoidcysten der Befund von Zähnen erwähnt (Bergmann, Gordon, Harres).

Eine Sonderstellung nehmen diejenigen geschwulstartigen Mißbildungen des Mediastinum ein, die keine Derivate der Haut zeigen. Es sind dies die drei Fälle von Joël, Helbing und Rolleston. Der erstere enthielt Cysten mit gallertartigem Inhalt, ausgekleidet von flimmerndem Zylinderepithel, Schleimdrüsen, glatte Muskulatur, Knorpel und viel lymphoides Gewebe. Genetisch glaubte Joël sein Teratom auf aberrierte Keime des Bronchialbaumes zurückführen zu müssen, in eine Zeit, in der Herz und Pleurahöhlen miteinander noch in offener Verbindung standen, da interessanterweise der Sitz dieses Teratoms innerhalb des Herzbeutels gelegen war.

Das Teratom in Helbings Fall enthielt vorwiegend quergestreifte Muskulatur, Knorpel, lymphoides Gewebe und verhältnismäßig spärlich Cysten, von kubischem Epithel ausgekleidet. Helbing faßte es als teratoide Bildung auf, die sich an Stelle der linken Lunge, die vollkommen fehlte, entwickelt hatte, und benennt sie wegen des Überwiegens der quergestreiften Muskulatur in ihrem Aufbau als Rhabdomyom. Auch genetisch dürfte dieser Fall wohl eher als zweikeimblättrige Mischgeschwulst, gesondert von den eigentlichen dreikeimblättrigen Teratomen des Mediastinum zu betrachten sein.

Das gleiche gilt auch für den von Rolleston beschriebenen Fall, der im besonderen sarkomatöse Bildungen zeigt. Auf Grund seiner histologischen Ähnlichkeit mit den Mischtumoren der Parotis wird er vom Autor als maligne Mischgeschwulst entodermaler Herkunft gedeutet. Rollestons Fall stellt eine Kombination eines cystischen Adenoms mit einem hämorrhagischen Chondrosarkom dar; er besteht aus Drüsen 
mit Becherzellen, glatter Muskulatur und bronchusähnlichen Strukturen und andernteils aus sarkomatösen, spindeligen, ovalen und ganglienzellenähnlichen Zellformen.

Abgesehen von diesen drei zuletzt genannten Fällen enthalten alle übrigen Mediastinalteratome, die demnach die kleinere Hälfte der bisher bekannten Mediastinalteratome ausmachen, Abkömmlinge aller drei Keimblätter und gehören mithin zur Gruppe der sogenannten komplizierten Dermoidcysten. Viele von ihnen sind histologisch genau untersucht, so die bekannten Fälle von Virchow, Török, Eckehorn, Dangschat, Madelung, Sieber, Ceelen, Pohl, Kleinschmidt, Ritchie. Thre Zusammensetzung ist eine recht vielartige. Sie enthalten meistens Cysten mit verschiedenartiger Epithelauskleidung und zeigen neben Haut und ihren Anhangsgebilden nervöses Gewebe, Bildungen vom Aussehen des Respirations- und Darmtraktes, überhaupt verschiedene entodermale Bildungen (Pankreasgewebe), ja sogar auch noch besondere ektodermale Derivate von Ähnlichkeit mit Chorionepitheliomen. Gehalt an Knochen und Zähnen wird vielfach erwähnt. Insbesondere Török beschreibt bei seinem Fall eine Kopfanlage mit Haarschopf, Lippe und Darm.

In wenigen Fällen dieser letzten Gruppe ist Malignität in Form teils sarkomatöser, teils carcinomatöser Bildungen mit Metastasierung ähnlich gebauter Knoten in Lungen, Leber und Milz beschrieben worden (Virchow, Jores, Ceelen und Ritchie).

Die Entstehung der Mediastinalteratome wurde anfänglich von den Autoren, die sich zuerst mit dieser Frage befaßten, mit der Zersprengungstheorie zu erklären versucht, nach der genau beim Schluß der vorderen Brustwand Teile des äußeren Keimblattes nach innen und Teile des Respirationstraktes nach außen verlagert werden sollen. Doch wurde ihre Unhaltbarkeit gleich von a.lem Anfang an erkannt, da niemals irgendwelche Spuren dieser angenommenen Zersprengung am Thorax oder an den Organen der Brusthöhle zu finden waren.

Nach Marchand, Pflanz und anderen wurden die Mediastinalteratome als branchiogene Organe aufgefaßt, $\mathrm{d}$. h. sie wurden genetisch in $\mathrm{Zu}$ sammenhang gebracht mit der Entstehung des Thymus bzw. der Thyreoidea. Pflanz nahm insbesondere für die Fälle von Marchand und Pinders den von der III. äußeren Kiementasche abgeschnürten Fundus praecervicalis, den ektodermalen Epithelkörper, der normalerweise obliteriert, als Ausgangsort der Mediastinalteratome an. In analoger Weise wurde auf die IV. äußere Schlundtasche im Zusammenhang mit der Entstehung der Schilddrüse für den Fall von Collenberg-Waldeyer hingewiesen. Die genannten Autoren hatten in einem Strang, der von der Hinterfläche des Mediastinalteratoms gegen die obere Brustapertur bis zur Thyreoidea verlief, Thymus- bzw. Schilddrüsengewebe (Collenberg- 
Waldeyer) gefunden und glaubten damit ihre Auffassung der Mediastinalteratome als branchiogener Bildungen erwiesen. Dementsprechend wurden die verschiedenen Befunde von cutanem oder mukösem Epithel von der äußeren oder inneren Schlundtasche abgeleitet.

Abgesehen davon, daß es nach Rautenberg fraglich erscheint, ob das Epithel der Kiemenspalte imstande ist, äußere Haut zu bilden, so reicht die Ansicht von Pflanz nicht aus, die sogenannten komplizierten Dermoideysten zu erklären. Dangschat läßt die Anschauung von Pflanz, nach der die Mediastinalteratome branchiogene Organe, also monogerminale autochthone Bildungen sind, nur für die sogenannten einfachen Dermoidcysten gelten. Die sogenannten komplizierten Dermoidcysten faßt Dangschat als ,,inkludierte Föten“ auf, als bigerminale Bildungen, die durch die Kiemenspalten oder vor Schluß der Thoraxhöhle in die Brustregion eingeschlossen werden und höchstens sekundär in Beziehung zum Thymus oder zur Thyreoidea geraten. In gleicher Weise glaubt Török für sein Mediastinalteratom eine zweite Keimanlage annehmen zu müssen, die vom Autositen umwachsen und in die Brusthöhle eingeschlossen wird. Auch Askanazy, der auf der XI. Tagung der deutschen pathologischen Gesellschaft im Jahre 1907 sehr eingehend die Frage der Teratomgenese erörterte, führt die Mediastinalteratome auf einen fast eiwertigen Keim zurück (wahrscheinlich eine Blastomere), der durch die Kiemenspalten in den Brustraum gelangen soll. Askanazy hält die Mediastinalteratome für bigerminale Bildungen und zählt sie zu den koätanen Teratomen, die durch ein mit dem des Trägers synchrones Wachstum aus einem fast eiwertigen Keim entstanden sind - im Gegensatz zu den malignen embryonalen Teratomen, die nach Askanazy durch heterochrones geschwulstmäßiges Wachstum des im übrigen gleichartigen Keimes entstehen.

Borst führt die Teratome der Brusthöhle auf generative Zellen (Keimzellen) zurück, die ursprünglich einen großen Bezirk der Leibeshöhle auskleiden, hier pathologischerweise liegen bleiben und selbständig zu wuchern beginnen.

Eine überaus einheitliche Erklärung der Teratomgenese versucht Budde in allerjüngster Zeit in seinem Beitrag zum Teratomproblem zu geben, die schlechtweg für alle dreikeimblättrigen Teratome in den verschiedenen Körpergegenden gilt. Als das charakteristische Zeichen einer bigerminalen Bildung fordert Budde die Segmentierung der Körperachse, die der Gastrulation zeitlich nachfolgt; mit der Gastrulation ist für Budde der Begriff Embryo, als selbständiger Keim, unzertrennlich verbunden. Die Vorstellung der Inclusio foetalis, d. h. der Umwachsung einer Embryonalanlage durch eine andere, hält Budde aus rein formalen Bedenken auf Grund der neuesten Kenntnisse über die Entwicklungsanfänge des menschlichen Embryos für unrichtig. Aus dem gleichen 
Grunde hält Budde auch die Hypothese des befruchteten Richtungskörperchens sowie die Marchand-Bonnetsche Auffassung der ausgeschalteten Blastomere für unannehmbar, wonach Richtungskörperchen wie Blastomere nach Einschluß in den Körper des normal sich entwickelnden Embryos zum Teratom heranwachsen sollen. Da alle Teratome des Mediastinum einen metameren Aufbau vermissen lassen und unter der Haut, d. h. also in der Brusthöhle des Autositen liegen - im Gegensatz zu den parasitischen Doppelbildungen, die wie z. B. der Thoracopagus als äußerer Anhang auf der Haut des Autositen erscheinen -, so sind nach Budde alle dreikeimblättrigen Teratome des Mediastinums monogerminale, autochthone Bildungen. Und zwar führt er sie genetisch auf ausgeschaltete Zellkomplexe des Urmundes zurück, die dann selbständig wuchern und demnach nicht koätane, sondern postätane Bildungen darstellen.

Der mediale Sitz und die Lage der Teratome vor der Wirbelsäule, am Ort des ,wandernden Urmundes", gilt für Budde auch als Stütze seiner einheitlichen Auffassung.

Je nach dem Differenzierungsgrad der Gewebe teilt Budde die dreikeimblättrigen Teratome in embryomorphe und in embryoide ein.

Alle nicht dreikeimblättrigen Bildungen faßt Budde als Hamartome, als örtliche Fehlbildungen auf, deren teratogenetische Terminationsperiode in einem späteren Zeitpunkt liegt.

Wenn auch vielfach die histologische Untersuchung sogenannter einfacher Dermoideysten nur ektodermales und mesenchymales Gewebe ergab, so war sie doch nicht eingehend genug, um etwa entodermale Bildungen ausschließen zu können. In Lage, Gewebsreife und zum Teil auch im Aufbau zeigen sie doch ein den sogenannten komplizierten Dermoidcysten ähnliches Verhalten, so daß man sie mit einer gewissen Reserve auch genetisch ähnlich beurteilen darf: also als embryoide Teratome im Sinne Buddes.

Jedenfalls muß eine genaue histologische Untersuchung auf Dreikeimblättrigkeit vorausgehen. Erst dann ist es erlaubt, im Falle des Fehlens von Bildungen eines Keimblattes, eine einseitige Differenzierung eines multipotenten Keimes anzunehmen, der im übrigen Bildungen aller dreier Keimblätter zu erzeugen vermag.

\section{Eigener Fall.}

In dem von mir beobachteten Fall handelte es sich um einen 41 jähr. Mann, der von der I. medizinischen Klinik (Prof. R. Schmidt) mit der klinischen Diagnose eines Aneurysma aortae descendent. am 14. II. 1920 zur Obduktion kam.

Auszug aus der Krankengeschichte: Vor 19 und vor 12 Jahren in psychiatrischer Behandlung wegen Gesichts- und Gehörshalluzinationen. Vor 6 Jahren zum ersten- 
mal Kurzatmigkeit, Stechen in der Herzgegend. Vom Arzt wurde schon damals „Verdunkelung“ auf der linken Brustseite festgestellt. Später Besserung der Beschwerden. Seit vielen Jahren Husten mit schleimigem Auswurf. Vier Monate ante exitum neverliches Einsetzen von Schmerzen und Schluckbeschwerden; es traten Ödeme auf, der Harn wurde spärlich. Bei Ruhelage schwanden die Ödeme. Rückenlage bei erhöhtem Oberkörper und rechter Seitenlage wurde nicht ertragen.

Kein Gelenkrheumatismus; keine luetische Infektion; keine körperlichen Anstrengungen.

Bei der Aufnahme: Puls 96, beiderseits gleich stark; keine respiratorische Arrhythmie; kein Pulsus paradoxus; Atmungsfrequenz 28; seit 2 Monaten Hinterhauptsschmerzen gegen Morgen.

Status praesens: Blasses Aussehen, untersetzte Statur, kxäftiger Knochenbau. Kyphotische Vorwölbung im Bereiche der unteren Brustwirbel. Ödem der Extremitäten ad sacrum, Anasarka an Brust und Bauch. Im Abdomen keine freie Flüssigkeit nachweisbar. Cyanose an Fingern, Zehen und im Gesicht. Inspektion und Palpation der Brust ergibt nichts Besonderes; Spitzenton nicht tastbar. Herzdämpfung reicht nach rechts bis zum rechten Sternalrand, nach links bis zur Mamillarlinie und geht nach oben in einen Dämpfungsbezirk über, der rom. linken Sternalrand an die ganze linke obere Brustseite umfaßt und sich gegen die Axilla ein wenig aufhellt, während infraclavicular bis zur III. Rippe massive Dämpfung besteht. Oliver-Cardarellisches Symptom negativ. Herztöne dumpf, aber rein. Hinten über der linken Iungenspitze etwas verkürzter Perkussionsschall. Über den ganzen Lungen diffus, besonders basal feuchte und trockne Rasselgeräusche, Giemen und Schnurren im In- und Exspirium. Abdomen anfgetrieben; unterer Leberrand in Nabelhöhe tastbar, bei Palpation schmerzhaft. Im übrigen: Wulstung der Rachenschleimhaut, Lingua scrotalis, Andeutung von Synophrys und Hallux valgus. Links eine überzählige Brustwarze unterhalb der Mamilla; am Rücken vereinzelte Naevi pilosi.

Im Verlauf der Krankheit nehmen Cyanose und Dyspnöe ständig zu. Leber wird 2 Querfinger unter dem Nabel tastbar.

Röntgenbefund: Links dem Herzen aufsitzend ein kugelrunder Schatten, anscheinend Aneurysma.

Während ophthalmoskopischer Untersuchung plötzlich einsetzende Verschlimmerung, starke Dyspnöe, Cyanose, elender Puls. Eine Stunde darauf Exitus.

Selation: Eine über mannsfaustgroße Dermoidcyste im vorderen Mediastinum mit Verwachsung der Cyste mit dem Herzbeutel und der medialen Fläche des linken Lungenoberlappens. Eine bohnengroße und eine erbsengroße Dermoidcyste (?) im interlobären Spalt zwischen linkem Ober- und Unterlappen. Fast vollständige Kompressionsatelektase des linken Oberlappens. Atelektasen geringeren Grades im linken Unterlappen. Fast totale adhäsive Pleuritis der linken Lunge. Partielle interlobäre Pleuritis rechts. Ein erbsengroßer schwieliger Herd mit Verkalkung nach Tuberkulose in der Spitze des rechten Oberlappens mit narbiger Einziehung der Oberfläche und partiell adhäsiver Pleuritis über der Spitze. Ein kleinbohnengroßer abgekapselter kreidig-kalkiger Herd-nach Tuberkulose in der Pleura der basalen. Fläche des linken Unterlappens. Verkreidung und partielle Verkalkung der beiderseitigen unteren tracheobronchialen Lymphknoten. 
Reste von Endokarditis mit geringer Verwachsung der Aortenklappen und Fensterung der Klappen. Umschriebene adhäsive Perikarditis im Bereich der Basis des Herzens und Sehnenflecke an der hinteren und vorderen Fläche des Herzens. Exzentrische Hypertrophie des rechten Ventrikels.

Chronischer Stauungsmilztumor. Staungsleber mit Atrophie und umschriebener adhäsiver Perihepatitis. Stauungsnieren. Stauungskatarrh des Magendarmtraktes. Ödem und Hyperämie des Gehirns. Chronischer Hydrocephalus internus. Chronische Leptomeningitis über dem vorderen Anteil der Konvexität. Hydrothorax, Hydroperikard, Hydroascites. Ödem der unteren Extremitäten.

Schwielen im Mesenterium. Verkalkung eines mesenterialen Lymphknotens.

Embryonale Lappung der Nieren. Abnorme Kerbung der Milz. Lipoidreiche Nebennieren.

Nach dem Abheben des Sternums findet sich ein über mannsfaustgroßer runder Tumor, der dem Herzbeutel aufliegt und auf den ersten Anblick wie ein zweites Herz imponiert. Die Kapsel des Tumors ist mit dem Sternum fest verwachsen. Der Tumor liegt über der Herzbasis und der Arteria pulmonalis auf. Er ist einerseits mit dem Herzbeutel, andererseits mit der medialen Fläche des linken Lungenoberlappens fest verwachsen und reicht mit seiner oberen Kuppe bis zum Arous aortae. Er liegt demnach im vorderen mediastinalen Gewebe zwischen Aorta ascendens, Arcus aortae, Herzbeutel und linkem Lungenoberlappen. Die Oberfläche des Tumors zeigt Auflagerungen von Fettgewebe. Seine Wand ist dick und teilweise hart. Der Tumor erweist sich nach dem Einschneiden als eine Cyste, die neben geringen Mengen eiterähnlicher trüber Flüssigkeit reichlich eine dickliche, pomadenartige, fast ockergelbe, von zahlreichen feinen Haaren durchsetzte Masse enthält. Die Innenfläche der Cyste ist uneben und zeigt zahlreiche platte kalkige Einlagerungen verschiedener Größe. Die Farbe der Innenfläche ist teils graulichweiß und zeigt hier hyalinartigen Glanz, teils aber, besonders in der linken Hälfte, stark gerötet.

Im linken unteren Teil der hinteren Wand findet sich eine etwas dunkler gefärbte, nicht gleichmäßig scharf begrenzte, fast gleichseitig dreieckige Partie von $2^{1 / 2} \mathrm{~cm}$ Seitenlänge, die weich, leicht erhaben, von schwarzen Haaren durchsetzt ist und wie Kopfhaut aussieht. Die vordere Wand ist in ihrer oberen Hälfte durch eine ungefähr $1 \mathrm{~cm}$ dicke Schicht verbreitert, die wie Muskulatur aussieht and auf der Schnittfläche einen fast haselnußgroßen und einen etwas kleineren und schmäleren Hohlraum zeigt, an deren Innenfläche verschieden große trabekelähnliche Wülste sichtbar sind. In der hinteren Wand dieser verdickten Partie ist eingelagert ein spangenartiges Knochengebilde mit einem kürzeren Schenkel von $1 \mathrm{~cm}$ und einem längeren von $2 \mathrm{~cm}$, das lumenwärts zwei etwa erbsengroße zahnartige Höcker zeigt, die wohl die Wilmssche Zotte darstellen. Am Ende des längeren Schenkels erscheint die Innenfläche in über linsengroßer Ausdehnung weißlich, wie chagriniert und von einzelnen Haaren durchsetzt.

Chemischer Befund: Der gelbe Farbstoff wurde auf Grund der Untersuchung im medizinisch-chemischen Institut (Prof. Zeynek) als zu den Luteinfarbstoffen zugehörig erkannt. 
Histologischer Befund: Es wurden zahlreiche Stücke aus verschiedenen Partien der Cystenwand nach Formolfixierung, teilweise nach Entkalkung in Gefrierund Paraffinschnitten untersucht. Die dabei verwendeten Färbungen waren: Hämalaun-Eosin, van Gieson, Kresolfuchsin, Perls Berlinerblaureaktion.

Die Cystenwand besteht größtenteils aus kollagenem Bindegewebe, das an vielen Stellen ausgedehnte hyaline Entartung und vielfach Verkalkung zeigt; dazwischen sieht man stellenweise markhaltigen $K$ nochen und mehr inselförmig hyaline Knorpelmassen mit geringer Verkalkung. Die Verkalkung ist in dem größten Teil der Cystenwand eine sehr hochgradige und ist in den innersten Schichten am stärksten ausgeprägt in Form von breiten Spangen und Schollen. Daneben sieht man in einzelnen Bindegewebsfasern auch ganz geringe Verkalkung. Elastische Fasern sind in allen Wandschichten vorhanden, in den äußeren besonders zahlreich, vielfach parallel zur Oberfläche verlaufend, ebenso in den inneren in der Umgebung der später zu besprechenden Drüsen und Cysten.

Die Anordnung der Bindegewebsfasern ist zwar größtenteils eine gleichmäßige, parallel zur Oberfläche, doch verflechten sie sich auch stellenweise in verschiedenen Richtungen miteinander zu einem teils sehr dichten, vielfach aber mehr lockeren Gefüge.

Eine epitheliale Auskleidung der Cyste ist nur noch stellenweise zu erkennen; sie wird durch ein mehrschichtiges Plattenepithel gebildet, dessen Zellen vielfach plattgedrückt erscheinen. Nur an wenigen Stellen sind sie gut erhalten, zeigen jedoch keine Verhornung. In einer dickeren Epithellage fällt ăn einer Stelle eine hornperlartige Anordnung der Epithelzellen auf. Pigment in den Basalzellen des auskleidenden Epithels habe ich nicht gefunden. Das geschichtete Pflasterepithel bildet auch einige meist seichte Einstiilpungen in die Cystenwand. Geschlossene Haufen und kleinere Zapfen von Plattenepithelien sind in nächster Nähe unter der epithelialen Auskleidung der Cyste, aber ohne erkennbaren Zusammenbang mit ihr vielfach $\mathrm{zu}$ sehen.

An den meisten Stellen der Cystenwand ist eine epitheliale Auskleidung nioht mehr zu erkennen. Sie ist hier wohl zugrunde gegangen. Teils liegen dann breite Kalkplatten, teils hyalines Bindegewebe dem Lumen der Cyste an, teils schmale Bänder eines noch nicht ganz verkalkten Gewebes, dessen Art, ob Epithel oder Bindegewebe, nicht mehr zu erschließen ist. An der einen Stelle der Innenwand, die makroskopisch durch ihre Behaarung und rote Farbe auffiel, erkennt man auch histologisch breite Massen von Blut und fettigem Detritus, sowie verkalktes Gewebe und dazwischen Quer- und Längsschnitte von Haaren, die jedoch keine Zellen mehr erkennen lassen, sondern nur aus grünlich-braunem Pigment bestehen.

An mehreren Stellen fand ich unter der innersten Schicht zahlreiche Drüsen, von hohem zylindrischem Epithel ausgekleidet. Diese Zellen gleichen auffallend den Basalzellen der Epidermis. An einer anderen Stelle, mehr gegen die mittleren Wandschichten zu, liegen deutliche Talgdrüsen, in ihrer nächsten Nähe größere Cysten von mehrschichtigem Plattenepithel ausgekleidet und zahlreiche Drüsen mit einreihigem hochkubischem Epithel, die wohl Schweißdrüsen sind, zwischen diesen Cysten und Drüsen breite Spangen hyalinen Knorpels.

Außer diesen größtenteils ektodermalen Bildungen sehen wir auch solche, die wir als entodermale auffassen dürfen. Es sind das teils rundliche, teils längliche unregelmäßige Cysten, die von einem Zylinderepithel ausgekleidet sind, das stellenweise als ein mehrreihiges Flimmerepithel erscheint, stellenweise aber nur einreihig und vielfach desquamiert ist. Es bildet deutliche papilläre Erhebungen und senkt sich mitunter zwischen diesen in Drüsenform in die äußeren Wandschichten der Cyste hinein. Einige dieser Papillen enthalten lymphfollikelähnliche 
Bildungen, im übrigen Gefäße und zahlreiche Plasmazellen. Das Epithel sitzt einer deutlichen Tunica propria auf. In einige dieser Papillen dringen glatte Muskelbuindel ein, die im übrigen in dichter und dünner Anordnung in der Wand und nächsten Umgebung dieser Cysten liegen. Die Muskelbündel haben einen verschiedenen Verlauf, erscheinen bald quer, bald schräg oder längs getroffen. Zwischen ihnen liegen einige isolierte Drüsen mit einreihigem Zylinderepithel. Auch sind Schleimdrüsen in der Umgebung einzelner solcher Cysten sicher erkennbar; ihr Epithel ist vielfach desquamiert, liegt dann im Cysteninhalt, in Schleim oder Detritusmassen. An der von Epithel entblößten Stelle ist die Wand der Cysten knapp unter der Tunica propria von Lymphocytenhaufen und Plasmazellen dicht infiltriert. Diese Infiltrate erwecken gemeinsam mit den deutlich hyperämischen Gefäßen unter dem Epithel den Eindruck eines entzündlichen Prozesses.

Glatte Muskulatur ist im übrigen in vielen Schnitten, besonders aus den Partien, die makroskopisch an Herzmuskulatur erinnerten, anzutreffen. Auffallend sind hier wiederum die mächtigen, oft knötchenförmigen Ansammlungen von Lymphocyten und die andern Ortes mehr diffuse Verteilung solcher, sowie von Fibroplasten und Plasmazellen zwischen den glatten Muskelbündeln und in den bindegewebigen Partien der Cystenwand. Es ist in den letzteren überhaupt auffallend viel, meist in breiten Längsreihen angeordnetes gefäßreiches lymphoides Gewebe zu sehen.

Altes Blutpigment ist stellenweise sehr reichlich in den äußeren und inneren Schichten der Cystenwand vorhanden, ebenso reichlich finden sich Blutgefäße. von mittlerem und größerem Kaliber. Die Wand der kleineren arteriellen Gefäße ist stark verdickt, die der größeren zeigt vielfach atheromatöse Veränderungen in der aufgelockerten breiten Intima und stellenweise beginnende Verkalkung der Media.

Fettgewebe ist in der Cystenwand, besonders in den äußeren Partien, reichlich enthalten. $\mathrm{Zu}$ erwähnen ist noch, daß stellenweise in lymphoidem Gewebe kleine regelmäßige Drüsen mit einreihigem kubischem Epithel und kolloidartigem Inhalt, inmitten von glatter Muskulatur, zu sehen sind, ohne Beziehung zum Cystenlumen oder zu einer kleineren Cyste innerhalb der Wand.

Als eigenartigen Befund möchte ich hervorheben, daß ich an einer, etwa der Größe einer kleinen Erbse entsprechenden Stelle, inmitten von lymphoidem Grewebe solide und drüsenartige Komplexe von epithelialen Zellen fand, die nach ihrer Anordnung und nach ihrem Aufbau eine maligne Wucherung darstellen. Die Zellen sind teils kubisch, teils zylindrisch, zeigen stellenweise deutliche Atypie und sind zum Teil verfettet. Wo sie sich zu Drüsen anordnen, ist in deren Lumen eine fädig geronnene schleimähnliche Masse mit desquamierten Epithelien zu sehen. Diese augenscheinlich carcinomatösen Bildungen sind jedoch nur im Bereiche dieses mehr oder weniger umschriebenen lymphoiden, von einigen hyalinen Bindegewebsfasern durchzogenen Gewebes zu sehen; ein Übergreifen auf die Umgebung hat nicht statt. Die soliden Komplexe und die drüsigen Bildungen hängen vielfach durch schmale epitheliale Zellstränge miteinander zusammen, wodurch vorwiegend das Bild einer malignen Wucherung in Form von zapfenartigen Fortsätzen bedingt ist. Doch sind außerdem auch isoliert liegende kleine und größere solcher Zellnester zu sehen.

Normale Drüsen habe ich an dieser umschriebenen Stelle nirgends gefunden; selbst wenn einzelne stellenweise auch ein schönes einreihiges Zylinderepithel zeigen, so ist immer an irgendeiner anderen, wenn auch nur kleinen umschriebenen Stelle eine deutliche Vermehrung der Zellagen und ein Hineinwuchern des Drüsenepithels in das umgebende lymphoide Gewebe zu beobachten.

Das mit der Cystenwand verwachsene Lungengewebe zeigt Atelektasen in mäßigem Grade und Verdickung des Interstitiums. Das anthrakotische Pigment 
der Lunge ist auch in den äußeren und mittleren Schichten der Cystenwand anzutreffen; an einer Stelle fand ich es sogar in der innersten Schichte im Bindegewebe, das hier geringe Verkalkung zeigt.

Die histologische Untersuchung der beiden kleinen zwischen linkem Oberlappen und Unterlappen gelegenen Cysten ergab bei beiden eine vollkommen hyalin degenerierte bindegewebige Wand, teilweise mit Verkalkung ihrer innersten Schicht. Die Wand der kleineren Cyste ist in höherem Grade verkalkt. Eine epitheliale Auskleidung ist bei keiner von beiden zu erkennen. Auf der Innenfläche liegen verkalkte körnige Massen, die wohl zum Teil Zelltrümmer darstellen, doch läßt sich die Herkunft dieser Zellen nicht mehr erschließen. Aus dem dermoidkreiähnlichen Inhalt, der dem der großen Cyste glich, möchte ich annehmen, daß beide Fehlbildungen darstellen, denen genetisch ektodermales, hautbildendes Gewebe zugrunde liegt. Etwas Bestimmtes über ihre Genese, insbesondere ihr genetisches Verhältnis zum großen Mediastinalteratom, läßt sich nicht sagen. Vielleicht sind sie auf die gleiche Entwicklungsstörung zurückzuführen, die dem Teratom zugrunde liegt, - es müßten dann ihre Keime zur selben Zeit und ungefähr am gleichen Ort ausgeschaltet worden sein, - oder auf eine teratogenetisch später datierende, mehr örtliche Fehlbildung. Thr Sitz und ihre Größe spricht für ein vom Teratom des Mediastinums unabhängig erfolgtes Wachstum.

In der Literatur wird nur ein einziger ähnlicher Fall unter den Teratomen der Brusthöhle von Sormani (zit. nach Pflanz) erwähnt. Sormani fand neben einer kindskopfgroßen Cyste mit cutaner Wand und mit Atherombrei und Haaren als Inhalt, die zu einem Drittel im linken Ober-, zu zwei Drittel im linken Unterlappen gelegen war, eine zweite kleinere Cyste von gleicher Beschaffenheit wie die große, aber vollständig getrennt von ihr im linken Oberlappen.

Die Epikrise unseres Falles ergibt ein etwa mannsfaustgroßes cystisches Teratom des vorderen Mediastinums, das klinisch jahrelang erhebliche Beschwerden verursachte. Anatomisch gehörte es in die Gruppe der dreikeimblättrigen Teratome oder der sogenannten komplizierten Dermoidcysten.

Es ist histologisch im allgemeinen typisch gebaut und zeigt $A b$ kömmlinge aller drei Keimblätter. Im besonderen sind seiner Wand stellenweise deutlich ausgeprägte entzündliche Veränderungen eigentümlich, wie sie sonst in der Literatur der Mediastinalteratome nicht erwähnt sind. Nur in einem von Dangschat mitgeteilten Fall war die Epidermisauskleidung des Teratoms stellenweise durch ein rotes Granulationsgewebe ersetzt.

Das Teratom zeigt reichlichen Gehalt an Hämosiderin, wie er in gleicher Weise auch in den beiden Fällen von Eckehorn erwähnt wird.

Als Besonderheit bietet dieser Fall eine umschriebene histologisch maligne epitheliale Wuchertung dar, wie sie in einem so geringen Umfang bei den im äbrigen großen Mediastinalteratomen noch nicht beschrieben wurde.

Sie war ein Zufallsbefund in einem etwa kleinerbsengroßen umschriebenen Bezirk. Die bekannten Fälle von Virchow, Jores und 
Ceelen betreffen auch maligne entartete Teratome der Brusthöhle mit Metastasenbildung, und zwar im Falle von Jores eine sarkomatöse, im Falle von Ceelen eine carcinomatöse Entartung, während Virchow in seinem oft zitierten Fall sarkomatöse und carcinomatöse Bildungen beschreibt.

Ich kann nun entgegen der in der Literatur herrschenden Auffassung Virchows Fall nicht als ein sogenanntes embryonales, blastomatöses Teratom im Sinne von Borst bezeichnen, da es neben unreifen embryonalen Bildungen auch solche von hoher Gewebsreife enthält, die nach Askanazy koätane Bildungen sind, wie wir sie in den echten Teratomen finden, so Cysten mit Dermoidbrei und Haaren. Es bestehen meines Erachtens nur graduelle, aber nicht wesentliche Unterschiede in bezug auf Größe und Ausdehnung der malignen Entartung im Teratom zwischen Virchows Fall und den Fällen von Jores und Ceelen. Man könnte im ersteren nach Borst auch eine Kombination von unreifen embryonalen mit reifen echten teratomatösen Bildungen erblicken. Doch müßBte man dann folgerichtig zwei ausgeschaltete Keime annehmen, deren verschiedenartiges und vor allem zeitlich getrenntes Wachstum zur Kombinationsmißbildungsgeschwulst führt. Ich halte es indessen für näherliegend, daß in Virchows Fall die mächtige blastomåtöse teratoide Bildung auf dem Boden eines schon bestehenden Teratoms entstanden ist.

Genetisch möchten wir unseren Fall so wie alle dreikeimblättrigen Teratome im Sinne Buddes deuten, dessen eingangs geschilderte Auffassung entwicklungsgeschichtlich derzeit am besten begründet erscheint, und ihn demnach in der Nomenklatur dieses Autors unter die embryoiden Teratome einreihen.

\section{Literaturverzeichnis.}

Aschoff, Lehrbuch der pathologisehen Anatomie. - Askanazy, Verhandl. d. path. Ges. 1907. - Borst, Verhandl. d. path. Ges. 1907. - Bergmann, Prager med. Wochenschr. 1898. - Budde, Beitr. z. allg. Path. u. pathol. Anat. 68. - Ceelen, Virchows Arch. f. pathol. Anat. u. Physiol. 20\%. - Dangschat, Beitr. z. klin. Chirurg. 38. - Eiselsberg, Arch. f. klin. Chirurg. \%1. - Ekehorn, Arch. f. klin. Chirurg. 56. - Garrè, Dtsch. med. Wochenschr. 1918. - Goebel und Ossig, Zentralbl. f. Chirurg. 1911. - Helbing Zentralbl. f. Path. 9. - Hertzell, Zentralbl. f. Chirurg. 1917. - Joël, Virchows Arch. f. pathol. Anat. u. Physiol. 81. - Jores, Virchows Arch. f. pathol. Anat. u. Physiol. 133. - Kaestle, Münch. med. Wochenschr. 1909. - Kleinschmidt, Münch. med. Wochenschr. 1920. - Madelung, Beitr. z. klin. Chirurg. 46. - Naegeli, Bruns' Beitr. z. klin. Chirurg. 110. - Pflanz, Zeitsehr. f. Heilk. 1\%. - Pohl, Dtsch. Zeitschr. f. Chirurg. 130. - Riegel, Virchows Arch. f. pathol. Anat. u. Physiol. 49. - Sieber, Virchows Arch. f. pathol. Anat. u. Physiol. 202. - Stilling, Virchows Arch. f. pathol. Anat. u. Phsyiol. 114. - Török, Zeitschr. f. Heilk. 21, Abt. f. Chirurg. - Virchow, Virchows Arch. f. pathol. Anat. u. Physiol. 53. - Westenryk, Prager med. Wochenschr. 25. - Wilms, Dtsch. Arch. f. klin. Yed. $\mathbf{5 5}$. 